\title{
Comparative study of three models of extra-articular distal humerus fracture osteosynthesis using the finite element method on an osteoporotic computational model.
}

\author{
Srećko Sabalića,*, Janoš Kodvanj ${ }^{\mathrm{b}}$, Arsen Pavićc \\ ${ }^{a}$ Trauma and General Surgeon, Sestre Milosrdnice University Hospital Center, University Hospital for Traumatology, Zagreb, Croatia \\ ${ }^{b}$ Faculty of Mechanical Engineering and Naval Architecture, University of Zagreb, Zagreb, Croatia \\ 'University Hospital Center Split, Split, Croatia
}

\section{KE Y W O R D S}

Distal humeral fracture

Extraarticular

Biomechanics

Finite element analysis

Osteoporosis

Plate fixation

Stability

Stiffness

\begin{abstract}
A B S T R A C T
Introduction: The biomechanical properties of extra-articular fractures of the distal humerus have not been researched sufficiently. The aim of the study was to examine three different models of osteosynthesis for extra-articular distal humerus fractures. Osteosynthesis with two parallel or perpendicular plates is a common method of osteosynthesis for those fractures. We wanted to examine the biomechanical performance of a newly designed Y plate, and compare it to the previously used osteosynthesis methods.

Materials and methods: On an osteoporotic computational model of the distal humerus, a 10mm gap was made, $25 \mathrm{~mm}$ above the olecranon fossa, and osteosynthesis was performed with the newly designed Y-shaped plate and with 3.5 reconstruction plates in parallel and perpendicular configuration. The numerical simulations in axial compression, bending and varus loading were conducted using the finite element method.

Results: On all models the largest displacements in the area of the fracture gap appear around the lower anterior edge. The parallel plate construct had the highest stiffness among the three plating techniques in axial compression. In bending and varus loading the construct with the newly designed plate had the highest stiffness, but in axial compression demonstrated the lowest. The parallel plate configurations had higher stiffness than the perpendicular ones in all three loading directions and the difference is most pronounced in axial compression.

Conclusion: The displacements that appeared in all three plating systems are minimal and within the limits that meet the requirements of sufficient biomechanical stability in the usual time for the healing of fractures in that region. The newly designed Y-shaped plate for extra-articular fractures of the distal humerus is a possible alternative to the usual method of osteosynthesis with two plates in the case of an extra-articular fracture of the distal humerus. Further biomechanical studies are needed for a decisive conclusion.
\end{abstract}

(c) 2013 Elsevier Ltd. All rights reserved.

\section{Introduction}

Although there is still some controversy regarding the treatment of extra-articular distal humerus fractures, many surgeons recommend surgery with plates and screws, ${ }^{1-7}$ while others give precedence to functional bracing, even in the case of a radial nerve lesion..$^{8,9}$ Robinson et al. ${ }^{1}$ reported a better healing response in operated patients with an extra-articular metaphyseal fracture of the distal humerus compared to patients treated non-operatively. Jawa et al. ${ }^{10}$ found a more significant loss of elbow and shoulder function in non-operated vs. operated patients, and concluded that operative treatment provides a

\footnotetext{
* Corresponding author at: Medveščak 85, 10000 Zagreb. Tel.: ++38598514381.

E-mail address: ssabalic@gmail.com (S. Sabalić).
}

quicker and more complete recovery, warning, however, that it also increases the risk of complications in the form of iatrogenic injuries of the radial nerve and wound infection.

The main objective in the management of these fractures is to re-establish alignment and obtain stable fixation in order to facilitate as soon as possible early range of motion, which is necessary for a good functional outcome., ${ }^{2,3}$

Distal humeral fractures have a relatively small incidence, but a large number of subtypes. ${ }^{9}$ No prospective randomized studies have been published on their outcomes, and the majority of the studies carried out were retrospective and involved a small number of patients..$^{10-23}$

Hence it is not possible to draw conclusions from them on the ideal configuration of the implant for stabilization of distal humerus fractures. 
There have been no studies dealing with fractures on the transition of the distal diaphysis into the distal humerus. In this region, no studies have been conducted using the finite element method.

The aim of this study was to preconfigure the $\mathrm{Y}$ reconstructive plate intended primarily for the treatment of intra-articular fractures of the distal humerus, and to convert it for extraarticular fractures of the distal humerus and distal humeral diaphysis, as well as to examine its biomechanical performance comparing it to the existing osteosynthesis methods with two reconstruction plates in perpendicular and parallel position using the finite element method. Our hypothesis was that the osteosynthesis with a novel Y plate would show at least the same stability of distal humerus fractures as well as the double plating systems with reconstruction plates in parallel and perpendicular configuration.

The finite element method is a highly valuable test method comparable in value to experimental biomechanical studies. ${ }^{24}$

\section{Materials and methods}

Computational simulations using the finite element method were performed with the models of the test and control groups under axial, lateral and bending load.

The test model was a construction with the newly designed titanium plate (Figure 1a) placed on the bone with 12 titanium screws, and the control models were parallel (Figure 1b) and perpendicular steel reconstruction plates (Figure 1c), with the arrangement of screws as shown in the figures. In both control models, two 3.5 reconstruction plates with 8 holes were placed, with 3 screws made of stainless steel on each side of the gap, 6 screws per plate.

The plates, screws, and the external and internal contours of the intact synthetic osteoporotic distal humerus (Synbone, Malans, Switzerland) were scanned with an optical 3D scanner (Atos III Triple Scan, GOM mbH, Germany). From these scans, the geometries were reconstructed using Geomagic Spark (3D Systems, USA) and GOM Inspect (GOM mbH, Germany) software packages. All screws were modeled without threads. A 10 $\mathrm{mm}$ long gap was placed on the computer model of the distal humerus, $25 \mathrm{~mm}$ above the olecranon fossa. Each model was 140 $\mathrm{mm}$ long and constrained at the proximal end. The computational simulations were performed using the finite element software Abaqus 6.10-1 (Dassault Systèmes, France).

Contact interactions using surface to surface finite sliding with a coefficient of friction of $0.3^{25}$ were defined between the bone and the plates. Tied constraints were applied between the screws and plates, as well as between the screws and the surrounding bone in all constructs.

The models were meshed with ten node quadratic tetrahedral elements (C3D10). The intact bone consisted of 407,699 elements, and each plate construct of approximately 800,000 elements. The element size was chosen based on a mesh convergence analysis of displacements and Mises stresses by using a model of the intact humerus.

Computational simulations were performed with loads on the specific part of the articular segment of the distal humerus, distally from the gap, following the principles from the latest biomechanical studies dealing with distal humerus fractures ${ }^{14,16-18}$, with the radial column being loaded with $60 \%$, and the ulnar column with $40 \%$ of the total load. The load and humerus positions were as follows: Axial load or anterior deflection in which the upper arm was placed at a flexion angle of about $5^{\circ}$ in relation to the longitudinal axis of the humeral diaphysis (Figure 2a); Bending or posterior deflection with the upper arm flexed at a $75^{\circ}$ angle in relation to the longitudinal a)

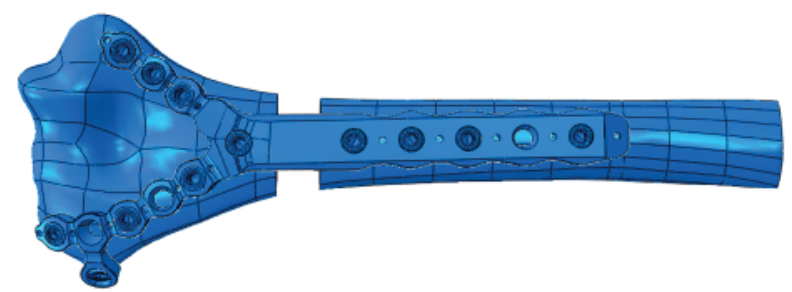

b)

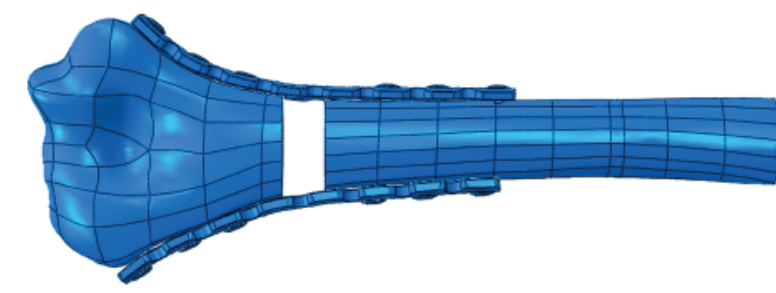

c)

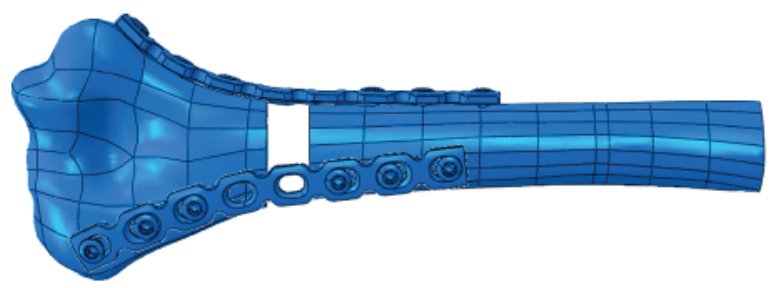

Fig. 1. Computational models: a) with newly designed Y-shaped plate, b) with parallel reconstruction plates, c) with perpendicular reconstruction plates.

axis of the humeral diaphysis (Figure 2b); Lateral or varus load in which the load is applied laterally on the radial condylar, with the humerus placed horizontally (Figure 2c).

In order to avoid the appearance of plastic deformations, in the axial direction the models were loaded with $200 \mathrm{~N}$, and in bending (posterior deflection and varus loading) with $30 \mathrm{~N}$. These loads were applied on the surfaces as shown in Figure 2.

Stainless steel and titanium alloy were assumed linear elastic, homogeneous and isotropic, with a Poisson's ratio of 0.3 and modulus of elasticity of 200 and $110 \mathrm{GPa}$, respectively. ${ }^{24,26}$

The Poisson's ratio for osteoporotic bone was set to be $0.4^{27}$ and the moduli of elasticity used for the cortical bone in axial compression, posterior deflection and varus loading were $3400 \mathrm{MPa}, 1150 \mathrm{MPa}$ and $660 \mathrm{MPa}$, respectively. As there was no information from the manufacturer about the bone material properties, these moduli of elasticity were determined by comparing linear elastic displacements obtained numerically by finite element analysis and experimentally on identical synthetic osteoporotic humeri.

\section{Results}

In all samples, maximum displacements appear on the distal articulation part more pronouncedly on the radial column, and are the largest in bending. Figure 3 shows the control group model with a perpendicular configuration of the reconstruction plates under lateral load in which the displacements are the largest.

Maximum displacements on the models of the test and control groups for all three loading directions are shown in Figure 4, and the stiffness under the same loading conditions in Figure 5. 

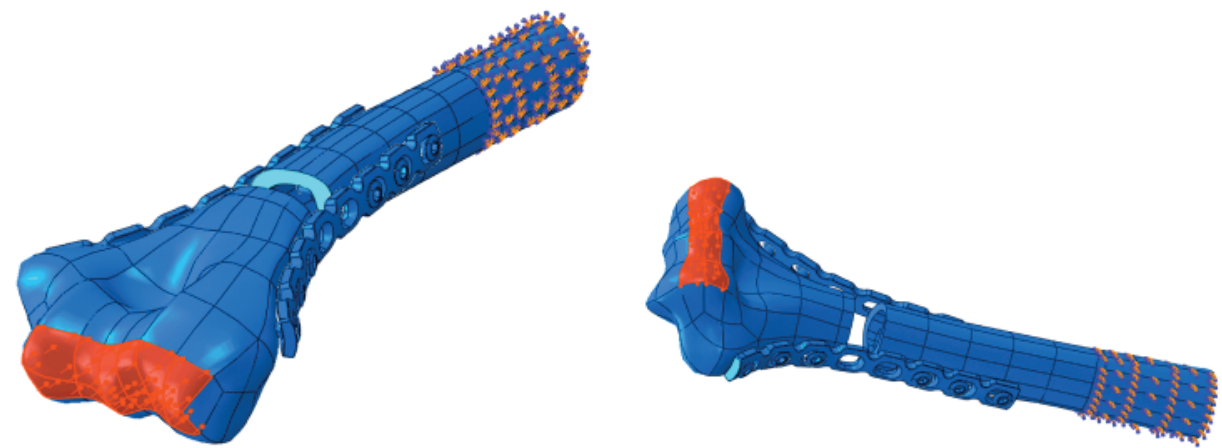

a)

b)

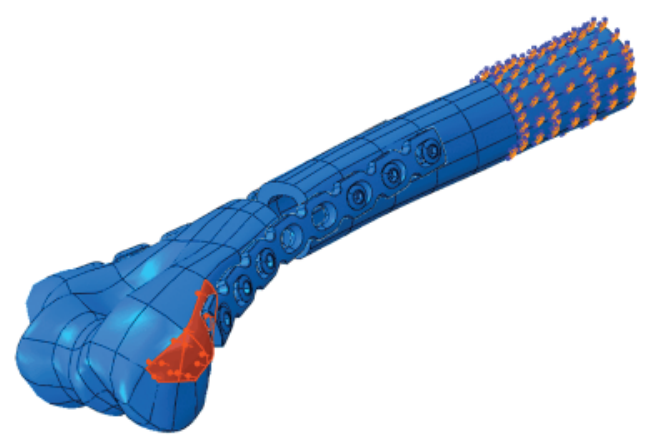

c)

Fig. 2. Loading conditions of computational models: a) axial load or anterior deflection, b) bending or posterior deflection, c) lateral or varus loading.
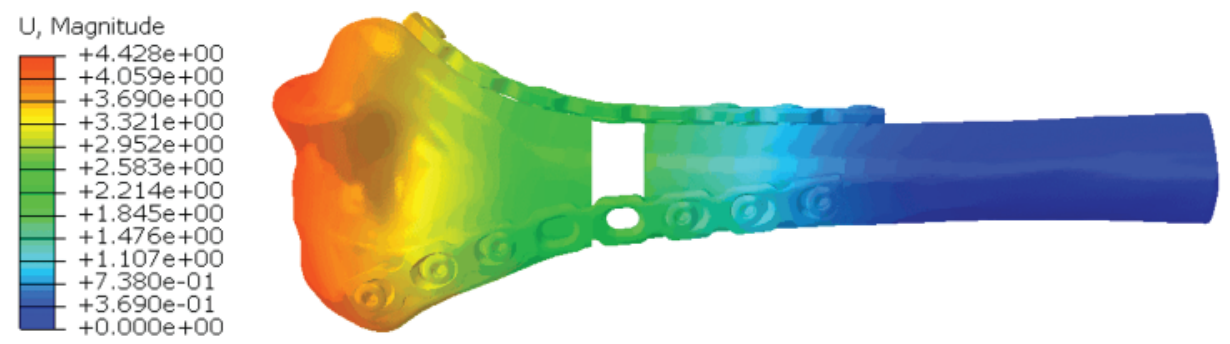

Fig. 3. Displacements magnitude on the model with a perpendicular configuration of reconstruction plates under lateral load.

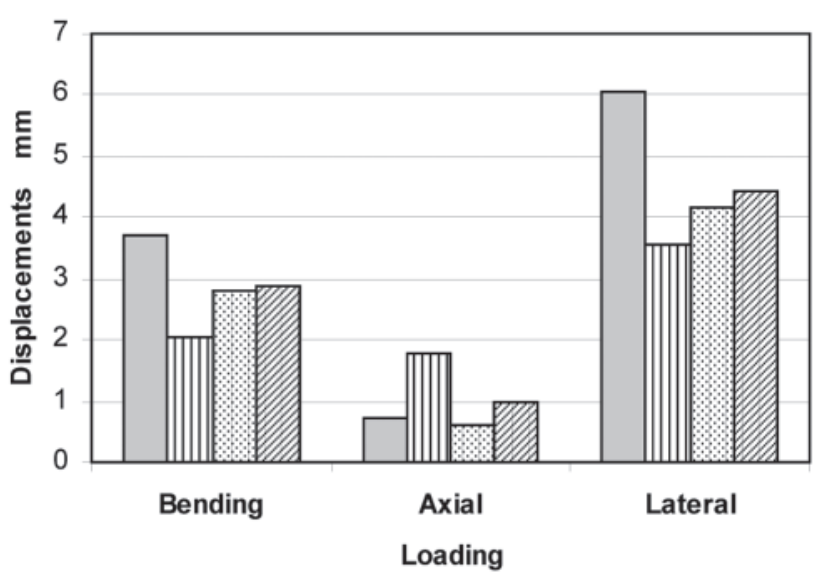

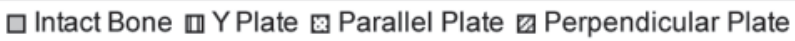

Fig. 4. Maximum displacements on the distal end of the test and control group models compared to intact bone.

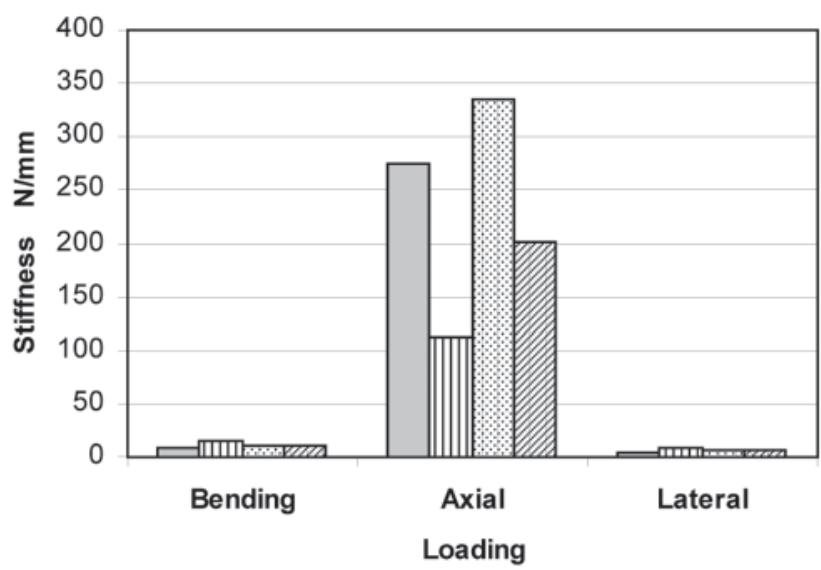

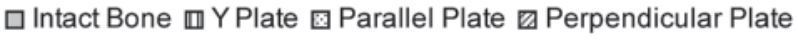

Fig. 5. Stiffness of the plate constructs and intact bone under different loads. 


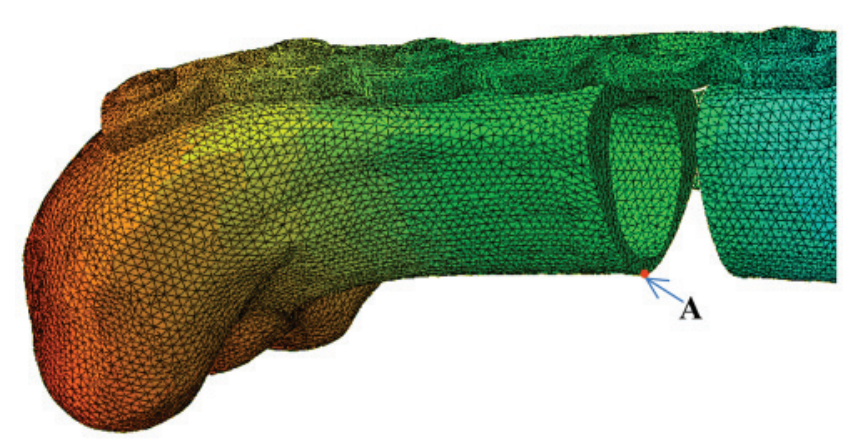

Fig. 6. Point A on the distal edge of the fracture gap.

Displacements on the fracture gap for all models were analyzed in point $A$ on the distal edge of the fracture gap (Figure 6 ). This point was selected because the analysis of displacements along the edge of the gap showed that the displacements in all models are the largest at that point and are shown in Figure 7.

Maximum von Mises stresses in the bone are smallest in the parallel configuration, and largest in the construct with the novel Y-shaped plate (Table 1). Under axial load in all models, the maximum stresses appear in the holes next to the fracture gap. Under bending and lateral load, the stresses are the greatest in the furthest proximal holes or at the clamped end, which was also confirmed by the results of preliminary studies.

In plates, similar as in the bones, maximum von Mises stresses are in the test plate (Table 1 ). The greatest difference in comparison with the control plates appears under axial load, while under bending and lateral load the differences are not significant.

\section{Discussion}

All of the previous biomechanical studies dealing with distal humeral fractures established the articular or immediate supracondylar level as the site of instability, which is imitated by the gap in the biomechanical model. ${ }^{11,13-18}$ No study to date has addressed the issue of biomechanical stability on the transition of the distal humeral diaphysis into the distal segment of the humerus.

The objective of the study was to examine the biomechanical properties of the newly constructed Y plate intended for the treatment of extra-articular fractures of the distal humerus, and to compare it with the existing common methods, with parallel or perpendicular plates. In the development of the idea of a new plate, several available facts were analyzed which could help improve the mechanical stability, as well as biomechanical studies and clinical papers with reference to complications, taking into account local anatomic conditions and common surgical procedures and approaches.

Loads were applied in the position of humeral flexion $\left(75^{\circ}\right)$ and extension $\left(5^{\circ}\right)$ with regard to the longitudinal humeral axis, as was done in the most recent studies. ${ }^{15-17}$ Loads applied in the study are within the physiological limits of loads in everyday activity of the postoperative rehabilitation period, and we avoided applying loads that would cause permanent plastic deformations on the implants. Furthermore, choosing the 10 $\mathrm{mm}$ gap length for this study was aimed at achieving extreme instability on the biomechanical model.

As computational simulations in this case served for orientation purposes, the final design of the novel Y plate was not insisted on in further research.

When selecting the appearance, primarily the arrangement of screws on the novel Y plate, effort was made to achieve a construction that would result in minimum displacements in

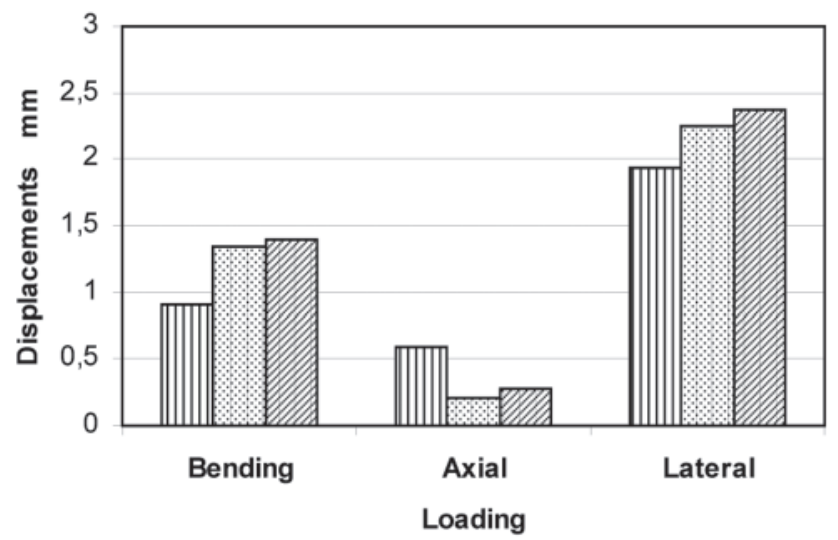

๓YPlate $\mathbb{0}$ Parallel Plate 0 Perpendicular Plate

Fig. 7. Displacements in point $A$ on the distal edge of the fracture gap.

Table 1

Maximum stresses in the bones and plates of the test and control models

\begin{tabular}{llccc}
\hline Load & & $\begin{array}{c}\text { Axial load } \\
(200 \mathrm{~N}) \\
\mathrm{MPa}\end{array}$ & $\begin{array}{c}\text { Bending } \\
(30 \mathrm{~N}) \\
\mathrm{MPa}\end{array}$ & $\begin{array}{c}\text { Varus load } \\
(30 \mathrm{~N}) \\
\mathrm{MPa}\end{array}$ \\
\hline Parallel & Bone & 10.86 & 13.98 & 14.12 \\
Perpendicular & Plate & 309.40 & 143.10 & 160.80 \\
& Bone & 18.48 & 14.95 & 15.19 \\
New plate & Plate & 280.40 & 152.40 & 193.80 \\
& Bone & 25.01 & 14.87 & 16.54 \\
& Plate & 567.40 & 189.00 & 189.00 \\
\hline
\end{tabular}

the direction of the load that causes greatest displacements, by comparing it with the constructions of the existing implants.

Under physiological conditions, most pronounced are the flexion ${ }^{15}$ and lateral ${ }^{12,18}$ load, while the axial load is considerably smaller. Significantly smaller displacements under axial load obtained in this study as well suggest a substantially lesser importance of that load in the overall evaluation of the construction's biomechanical stability, which is in line with previous studies ${ }^{17}$, although they were not conducted on the same level of the distal humerus.

As already mentioned, previous biomechanical studies were conducted on the intra-articular level, which renders the comparison with them more difficult. Those studies are very uneven: with regard to the number and types of models, methods and load directions, methods and precision of displacements measurements, and thus the conclusions derived from them are hardly mutually comparable..$^{11,13-18}$

Using the finite element method, attempts were made to find the optimal plate configuration which would improve its biomechanical properties, taking into account the specific anatomy of the distal humerus.

In addition to the bending and axial load, special attention was paid to the lateral or varus load as a previously neglected load with pronounced clinical significance. Namely, the force of gravity which acts on the long lever arm (the forearm) while the elbow is flexed and extended during activities apparently requiring minimal use, leads to repeated varus stresses in the elbow. As observed in previous studies, it is due to varus stresses that pseudoarthrosis of the distal humerus usually occurs in the region at the metaphyseal and supracondylar level of radial columns. ${ }^{12}$

It was precisely the lateral load on all models in this study that led to the largest displacements on the models, both on the articular surface and on the gap site. 
The largest displacements were found on the models in the gap area, in the test and both control models in all three load directions, in the region of the lower anterior edge, and on the anterior surface of the distal humerus model in the gap area. As expected, due to the $60-40 \%$ ratio of the load on the articular surface, the largest displacements on the load site occurred on the radial column. It was also observed that the displacements under bending and lateral loads were greater than the displacements under axial load. Consequently, displacements under bending and lateral loads to the radial condyle are considerably larger than under the axial load, and thus with a greater significance in the overall assessment of the structural stability of implants. The implant, therefore, has the task of neutralizing the forces that cause the greater shifts in the area. That piece of information conditioned the construction of a new implant that neutralizes the shifts in those load directions.

The fundamental possible objection to this study is the possibility in both control models, parallel and perpendicular, to extend the plates distally and thus increase the construction's biomechanical stability in those models. However, in that case the total number of screws in such models would increase significantly. Furthermore, due to the anatomy of the distal humerus, in reality it would be necessary to adapt the implants additionally to the complex anatomy of the distal humerus. Indeed, in clinical practice it is not common to place the plates and so many screws so distally in extra-articular fractures of the distal humerus. ${ }^{2-7,10,11}$

This study suggests that under physiological loads, which are common in the immediate postoperative period, the largest displacements on the joint surface are observed on the radial condyle, and the largest displacements in the gap area are observed in the front part. This information suggests that it is necessary, regardless of whether one or two plates are being placed, to achieve greater stability in the radial column area, which was also confirmed in clinical practice. ${ }^{2,4}$ The significance of lateral column stabilization is all the greater if we know that in the case of osteoporosis in the distal humerus region, it is most pronounced in the posterolateral segment. ${ }^{28,29}$ Moreover, it was found that the cortex in the medial column region is substantially stronger in comparison with the lateral column, both in the supra- and infracondylar region of the distal humerus. ${ }^{30}$

Significantly smaller displacements under axial load are comparable to those in the previous studies ${ }^{17}$, although they were not conducted at the same level of the distal humerus.

Axial fracture loading appears to be beneficial if applied in the early stage of fracture treatment. Namely, it is known that in delayed healing it can be beneficial to "dynamize" the fracture by compression (or traction) to assist healing. ${ }^{31}$ Therefore, axial instability that is more pronounced in the newly designed $\mathrm{Y}$ plate when compared to perpendicular or parallel plates could present an advantage in the healing of fractures in that region.

Simpler placement in comparison with the previously common osteosynthesis method with two plates is also a possible advantage of the preconfigured Y plate.

\section{Conclusion}

Under physiological loads, the radial column withstands greater loads, and displacements in the radial articulation surface are more pronounced. For that purpose, in extra-articular fractures of the distal humerus the implant should provide greater stability in the radial column area. In all three models (both the control and test models), the displacements occurring after all three loads are minimal and within the range that fulfills the requirements of sufficient biomechanical stability in the usual time for the healing of fractures in that region.
The newly designed $\mathrm{Y}$ plate for extra-articular fractures of the distal humerus is a possible alternative to the usual osteosynthesis method with two plates. A definitive conclusion would require biomechanical studies, either with a synthetic or a cadaveric model. Moreover, the impact of osteoporosis in the posterolateral segment of the distal humerus on the stability of the model, as well as the possible impact of LCP plates in osteoporosis requires additional biomechanical and clinical studies on larger samples, and measurements on biomechanical models performed with greater precision than was done in papers published to date.

\section{Conflict of interest}

All authors have no financial and personal relationships with other people or organisations that could inappropriately influence of the work.

\section{References}

1. Robinson CM, Hill RM, Jacobs N, Dall G, Court-Brown CM. Adult distal humeral metaphyseal fractures: epidemiology and results of treatment. $J$ Orthop Trauma 2003;17(1):38-47.

2. Levy JC, Kalandiak SP, Hutson JJ, et al. An alternative method of osteosynthesis for distal humeral shaft fractures. J Orthop Trauma 2005;19:43-47.

3. Darabos N, Bajs ID, Sabalic S, Pavic R, Darabos A, Cengic T. Elements for successful functional result after surgical treatment of intra-articular distal humeral fractures. Acta Clin Croat 2012;51(4):627-31.

4. Prasarn ML, Ahn J, Paul O, Morris EM, Kalandiak SP, Helfet DL, Lorich DG. Dual plating for fractures of the distal third of the humeral shaft. J Orthop Trauma 2011;25(1):57-63.

5. Mehne DK, Jupiter JB. Fractures of the distal humerus. In: Browner BD, Jupiter JB, Levine AM, Trafton PG, editors. Skeletal trauma. Volume 2. Philadelphia: Saunders; 1992. p.1146-76.

6. Chapman MW. Fractures and dislocations of the elbow and forearm. In Chapman MW, editor. Chapman's Orthopaedic Surgery. Philadelphia: Lipincott Williams \& Wilkins; 2001, p. 481-529.

7. Schatzker J. Fractures of the Distal End of the Humerus (13-A, B and C). In Schatzker J, Tile M, editors. The Rationale of Operative Fracture Care. Berlin, Heidelberg, New York: Springer; 2005, p. 103-121.

8. Sarmiento A, Horowitch A, Aboulafia A, et al. Functional bracing of comminuted, extra-articular fractures of the distal third of the humerus. JBone Joint Surg Br 1990;72:283-287.

9. Ekholm R, Ponzer S, Törnkvist H, Adami J, Tidermark J. The Holstein-Lewis humeral shaft fracture: aspects of radial nerve injury, primary treatment, and outcome. J Orthop Trauma 2008;22(10):693-7.

10. Jawa A, McCarty P, Doornberg J, Harris M, Ring D. Extra-Articular Distal-Third Diaphyseal Fractures of the Humerus. A Comparison of Functional Bracing and Plate Fixation. J Bone Joint Surg Am 2006;88(11):2343-7.

11. Tejwani NC, Murthy A, Park J, McLaurin TM, Egol KA, Kummer FJ. Fixation of extraarticular distal humerus fractures using one locking plate versus two reconstruction plates: a laboratory study. J Trauma 2009;66(3):795-9.

12. O'Driscoll SW. Optimizing stability in distal humeral fracture fixation. JShoulder Elbow Surg 2005;14:186S-94.

13. Korner J, Diederichs G, Arzdorf M, et al. A biomechanical evaluation of methods of distal humerus fracture fixation using locking compression plates versus conventional reconstruction plates. J Orthop Trauma 2004; 18:286-293.

14. Schwartz A, Oka R, Odell T, Mahar A. Biomechanical comparison of two different periarticular plating systems for stabilization of complex distal humerus fractures. Clin Biomech (Bristol, Avon) 2006;21:950-955.

15. Schuster I, Korner J, Arzdorf M, Schwieger K, Diederichs G, Linke B: Mechanical comparison in cadaver specimens of three different 90 - degree double-plate osteosyntheses for simulated C2-type distal humerus fractures with varying bone densities. J Orthop Trauma 2008;22:113-120.

16. Windolf M, Maza ER, Gueorguiev B, Braunstein V, Schwieger K. Treatment of distal humeral fractures using conventional implants. Biomechanical evaluation of a new implant configuration. BMC Musculoskelet Disord 2010;4(11):172.

17. Penzkofer R, Hungerer S, Wipf F, von Oldenburg G, Augat P. Anatomical plate configuration affects mechanical performance in distal humerus fractures. Clin Biomech (Bristol, Avon) 2010;25(10):972-8.

18. Zalavras CG, Vercillo MT, Jun BJ, Otarodifard K, Itamura JM, Lee TQ. Biomechanical evaluation of parallel versus orthogonal plate fixation of intra-articular distal humerus fractures. J Shoulder Elbow Sur 2011;20(1):12-20. 
19. Helfet DL, Kloen P, Anand N, Rosen HS. ORIF of delayed unions and nonunions of distal humeral fractures. Surgical technique. J Bone Joint Surg Am 2004;86(Suppl 1):18-29.

20. Luegmair M, Timofiev E, Chirpaz-Cerbat JM. Surgical treatment of AO type $\mathrm{C}$ distal humeral fractures: Internal fixation with a Y-shaped reconstruction (Lambda) plate. J Shoulder Elbow Surg 2008;17(1):113-20.

21. Helfet DL, Hotchkiss RN. Internal fixation of the distal humerus: a biomechanical comparison of methods. J Orthop Trauma 1990;4(3):260-4.

22. Schmidt-Horlohé K, Wilde P, Bonk A, Becker L, Hoffmann R. One-third tubular-hook-plate osteosynthesis for olecranon osteotomies in distal humerus type-C fractures: a preliminary report of results and complications. Injury 2012;43(3):295-300

23. Chen G, Liao Q, Luo W, Li K, Zhao Y, Zhong D. Triceps-sparing versus olecranon osteotomy for ORIF: analysis of 67 cases of intercondylar fractures of the distal humerus. Injury 2011;42(4):366-70.

24. Wieding J, Souffrant R, Fritsche A, Mittelmeier W, Bader R. Finite element analysis of osteosynthesis screw fixation in the bone stock: an appropriate method for automatic screw modelling. PLoS One 2012;7(3):e33776.

25. Chen AC-Y, Lin Y-H, Kuo H-N, Yu T-C, Sun M-T, Lin C-L. Design optimisation and experimental evaluation of dorsal double plating fixation for distal radius fracture. Injury 2013;44:527-534.
26. Plausinis D, Greaves C, Regan WD, Oxland TR. Ipsilateral shoulder and elbow replacements: On the risk of periprosthetic fracture. Clinical Biomechanics 2005;20:1055-1063.

27. Brown AT, Kohan L, Ben-Nissan Besim. Assessment by finite element analysis of the impact of osteoporosis and osteoarthritis on hip resurfacing. $5^{\text {th }}$ Australasian Congress on Applied Mechanics, ACAM 2007, Brisbane, Australia, 2007.

28. Korner J, Lill H, Müller LP, Rommens PM, Schneider E, Linke B. The LCPconcept in the operative treatment of distal humerus fractures biological, biomechanical and surgical aspects. Injury 2003;34(Suppl 2):B20-30.

29. Park SH, Kim SJ, Park BC, Suh KJ, Lee JY, Park CW, Shin IH, Jeon IH. Threedimensional osseous micro-architecture of the distal humerus: implications for internal fixation of osteoporotic fracture. J Shoulder Elbow Surg 2010;19(2):244-50.

30. Diederichs G, Issever AS, Greiner S, Linke B, Korner J. Three-dimensional distribution of trabecular bone density and cortical thickness in the distal humerus. J Shoulder Elbow Surg 2009;18(3):399-407.

31. Einhorn T. Enhancement of fracture-healing. J Bone Joint Surg 1995;77-A: 940-956. 